\title{
Implementing Routine Cognitive Screening of Older Adults in Primary Care: Process and Impact on Physician Behavior
}

\author{
Soo Borson, $M D^{1,2}$, James Scanlan, $P h D^{1,2}$, Jeffrey Hummel, $M D, M P H^{3,4}$, Kathy Gibbs, $R N^{4}$, \\ Mary Lessig, $B S^{1,2}$, and Elizabeth Zuhr, $B A^{1,2}$
}

'Department of Psychiatry and Behavioral Sciences, University of Washington, 1959 NE Pacific Street, Campus Box 356560, Seattle, WA 98195 , USA; ${ }^{2}$ Alzheimer's Disease Research Center, University of Washington, Seattle, WA, USA; ${ }^{3}$ University of Washington Physicians Network, University of Washington, Seattle, WA, USA; ${ }^{4}$ University of Washington Neighborhood Clinic, University of Washington, Seattle, WA, USA.

BACKGROUND: Early detection of cognitive impairment is a goal of high-quality geriatric medical care, but new approaches are needed to reduce rates of missed cases.

OBJECTIVE: To evaluate whether adding routine cognitive screening to primary care visits for older adults increases rates of dementia diagnosis, specialist referral, or prescribing of antidementia medications.

SETTING: Four primary care clinics in a universityaffiliated primary care network.

DESIGN: A quality improvement screening project and quasiexperimental comparison of 2 intervention clinics and 2 control clinics. The Mini-Cog was administered by medical assistants to intervention clinic patients aged 65+ years. Rates of dementia diagnoses, referrals, and medication prescribing were tracked over time using computerized administrative data.

RESULTS: Twenty-six medical assistants successfully screened $70 \%(n=524)$ of all eligible patients who made at least 1 clinic visit during the intervention period; $18 \%$ screened positive. There were no complaints about workflow interruption. Relative to baseline rates and control clinics, Mini-Cog screening was associated with increased dementia diagnoses, specialist referrals, and prescribing of cognitive enhancing medications. Patients without previous dementia indicators who had a positive Mini-Cog were more likely than all other patients to receive a new dementia diagnosis, specialty referral, or cognitive enhancing medication. However, relevant physician action occurred in only $17 \%$ of screen-positive patients. Responses were most related to the lowest Mini-Cog score level (0/5) and advanced age.

CONCLUSION: Mini-Cog screening by office staff is feasible in primary care practice and has measurable effects on physician behavior. However, new physician action relevant to dementia was likely to occur only when impairment was severe, and additional efforts are needed to help primary care physicians follow up appropriately on information suggesting cognitive impairment in older patients.

Received October 12, 2006

Revised March 8, 2007

Accepted March 26, 2007

Published online April 20, 2007
KEY WORDS: Mini-Cog; practice intervention; primary care; dementia screening; clinic intervention. DOI: $10.1007 / \mathrm{s} 11606-007-0202-8$ (C) 2007 Society of General Internal Medicine 2007;22:811-817

\section{BACKGROUND}

Underdiagnosis of dementia has been demonstrated in many studies. ${ }^{1-5}$ Dementia screening remains more controversial than screening for most other chronic conditions ${ }^{6,7}$ despite evidence that it could improve case finding. ${ }^{1,3,8,9}$ Practicing physicians acknowledge the importance of recognizing cognitive impairment, but important barriers, such as added visit time, still exist. ${ }^{1,8,10,11}$ The present study was designed as a practice intervention to test whether (1) a simple, brief, cognitive screen (the Mini-Cog) would be administered regularly and reliably by medical assistants in primary care practice and (2) implementation of screening would increase physician diagnoses of dementia, specialty referral, and/or prescription of antidementia medications.

\section{METHODS}

Setting and Patients. The University of Washington (UW) Physicians Neighborhood Clinics, a group of 8 primary care practices located in and around Seattle, provide over 240,000 primary care visits to more than 100,000 patients annually. All clinics in the network use the same administrative management information and electronic medical record system (EPICare). Four clinics were selected for this study, 2 pairs as intervention and control sites. Each pair included an urban and a suburban site and cared for about 1,000 older adults. Control clinic physicians were family practitioners $(n=10)$ or Internists $(n=10)$; intervention clinic physicians were family practitioners $(n=11)$, Internists $(n=6)$, or geriatricians $(n=2)$. Data on physician action outcomes by specialty were collected only for intervention clinics.

Patients were eligible for screening if they were seen for at least 1 clinic visit during the 12 -month planned intervention period, had not been previously screened, and were at least 65 years old at the time of their visit. No other selection criteria were built in to the study design. Older patients were eligible without regard to preexisting dementia diagnosis or treatment 
to maintain workflow and eliminate the need for time-intensive review of individual medical records in advance. Data on patient age, gender, and type of primary care physician (geriatrician vs General Internist or family physician), and dementia diagnoses, referrals, and medications were captured electronically for all clinics. Data were not collected on ethnicity, medical or psychiatric comorbidities, medication use other than cognitive enhancers, or health services utilization.

Implementing the Screening Process. The project was approved by the medical directors of the UW Neighborhood Clinic organization and each participating clinic, supervisors of intervention clinic medical assistants, and the UW Institutional Review Board. Brochures and flyers describing the project were posted in various locations including patient exam rooms of the intervention clinics. Medical and administrative staff of the intervention clinics were briefed about the rationale and methods of the study. Physicians were given brochures describing the study and choices they could make in response to a positive screen, including watch and wait, conduct a dementia evaluation in primary care, refer to a list of dementia specialists, and/or initiate a cognitive enhancing medication. A nurse in each intervention clinic functioned as the project champion and supervised screening by the MAs. One intervention clinic was selected for an initial trial period to troubleshoot the process before the protocol was extended to the second; control clinics received no brochures or contact from the research team other than to secure clinic directors' permission to use summarized clinical data on patients cared for at their sites.

MAs were trained in administration of the Mini-Cog, ${ }^{1,12,13}$ gave and scored it after completing vital signs (noting the reason the patient was not screened, if applicable), and recorded the result in the electronic medical record for review and further decision-making by the physician. MAs were instructed not to discuss screening results with the patient and to refer any questions about the process to the patient's physician. Scoring of the Mini-Cog by MAs was highly reliable (96\% concordance with research scoring).

Preventing Duplicate Screening. The electronic medical record utilized by the UW Physicians' Network Clinics did not allow electronic flagging of scheduled patients eligible for screening during each clinic session. The nurse champion therefore reviewed each day's schedule to identify those 65 years old and older, checked the record to see if the patient had previously been screened, and manually entered colored electronic dots next to patients' names, red signifying "needs screening” and green signifying "already screened."

Unanticipated Confounders. Intervention and control clinics proved to be imperfectly matched. By the time the intervention began, only test clinics had a geriatrician on staff ( 1 in each clinic) in addition to a much larger number of family practitioners and General Internists. Preliminary analyses showed differences between intervention and control clinic patients attributable to the presence or absence of geriatricians. Geriatricians' patients $(n=361)$ were less often women ( 55 vs $67 \%$ of nongeriatricians' patients, $P<0.001$ ) and were older (mean age $78.0 \pm 8.2$ years) than nongeriatricians' patients in both intervention ( $n=415$, mean age $74.0 \pm 7.3)$ and control $(n=$ 1,140 , mean age $74.1 \pm 6.9$ ) clinics. Outcome analyses therefore considered geriatricians' versus nongeriatricians' patients separately and covaried age and gender.

Process Issues: Early Termination. The screening project, planned to run for a full calendar year, was terminated early (at 9 months in the first intervention clinic and 3 months in the second) because of reassignment of nurse champions to different clinics in the network for reasons unrelated to this project. This administrative change disrupted MA supervision and the process of flagging patients eligible for screening, and rendered the total number of patients seen during the screening phase different from the total number seen during the calendar year.

\section{Evaluating Outcomes}

Uptake of Screening in the Intervention Period. All eligible patients making at least 1 intervention clinic visit during the active implementation phase of the project were included in this analysis $(n=748)$. Arriving patient lists for each clinic session were compared to completed Mini-Cog forms to determine the proportion of eligible patients actually screened, reasons for omission of screening (when reported), and the percentage with a positive screen.

Impact Measures. Impact was assessed using electronic capture of 3 dementia indicators, including the International Classification of Diseases, 9th edition, and the Diagnostic and Statistical Manual of Mental Disorders, 4th edition, dementia diagnoses, referrals to a dementia specialist, and/or prescription of a cognitive enhancing medication. Both an omnibus composite indicator (scored $0=$ none or $1=1$ or more indicators) and individual indicators were examined. Prescriptions for cognitive enhancing medications included all cholinesterase inhibitors (memantine was not available at the time of this study), coded as "ever prescribed" without regard to specific drug, dose, patient adherence, or ongoing use.

\section{Data Analyses}

Clinic Level Changes. Changes in individual dementia indicators (diagnosis, specialty referral, or antidementia medication prescription) over time were evaluated within and between intervention and control clinics, using repeated measures ANCOVA and post hoc tests of pooled data (Fig. 1). These analyses included intervention and control clinic patients who were seen in both baseline (2002-2003) and intervention (2003-2004) calendar years, and the time frame for assessing impact was 12 calendar months. In the intervention clinics, some patients were seen outside the active screening period because of the early termination of the project. Some were therefore not screened, although they would have been eligible had the project continued throughout the year.

New Physician Actions Related to Screening. The effects of passing or failing the Mini-Cog on change in dementia 
Impact of Screening Intervention on Physician Behavior ( $n=1916)$

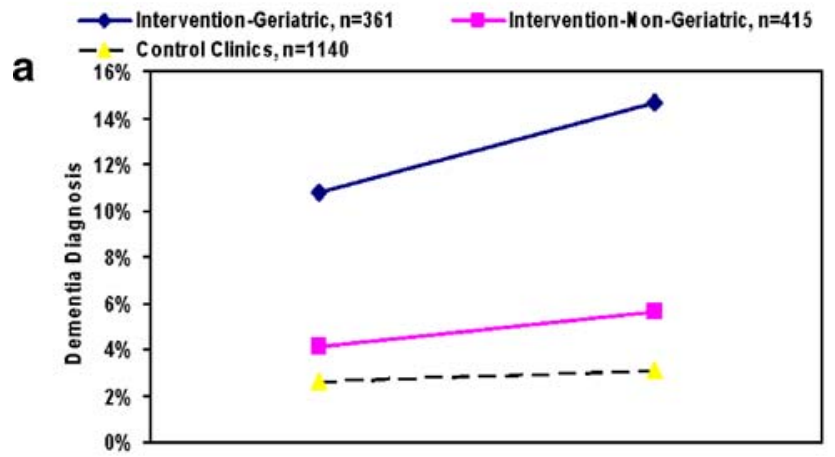

b

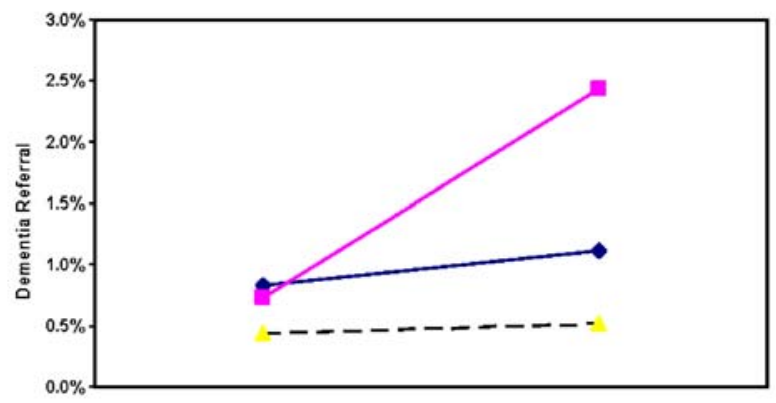

C

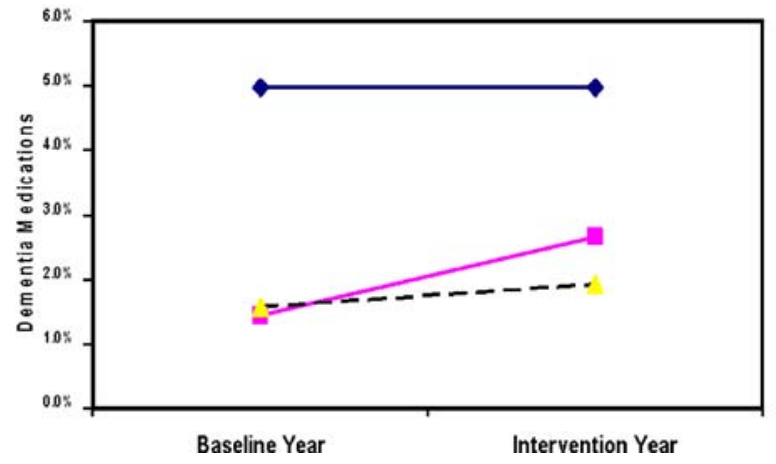

Figure 1. Impact of screening on physician behaviors. Dementia diagnoses increased $3.9 \%$ ( 39 to 53 cases) for geriatricians and $1.5 \%$ ( 17 to 23 ) for nongeriatricians in intervention clinics, and $0.5 \%$ (30 to 35 ) for control clinics. Dementia specialty referrals increased $0.3 \%$ ( 3 to 4 ) for geriatricians, $1.5 \%$ ( 3 to 10 ) for nongeriatricians, and $0.1 \%$ (5 to 6$)$ for control clinics. Dementia medication prescriptions did not change $(0 \%, 18$ in both years) for geriatricians, increased $1.2 \%$ ( 6 to 11 ) for nongeriatricians, and increased $0.35 \%$ (18 to 22 ) for control clinics

indicators were then evaluated. After excluding patients with baseline dementia indicators, the remaining patients were separated into 7 groups: control clinic patients and intervention clinic patients who were not screened, screened/passed, and screened/failed and were seen by nongeriatric specialty and geriatric specialty physicians $(3 \times 2$, see Fig. 2 for derivation of these groups and Fig. 3 for results). Differences between groups were tested with ANCOVA and post hoc tests.

Predictors of New Physician Actions. Within all screened intervention clinic patients without previous dementia indicators, logistic regressions were used to assess the relative influence of severity of cognitive impairment (Mini-Cog score), physician specialty, and patient age on new physician actions. All analyses were done using SPSS version 13.

\section{RESULTS}

\section{Implementation}

Quality Control and Uptake of Screening by Intervention Clinics. MAs and physicians made few complaints about the intervention, and their informally reported perception of workflow was not significantly affected by the use of the short screen adopted for this study and the related charting processes. Of all patients seen during the active screening period, regardless of whether they had been seen in the preceding year $(n=748), 524$ $(70 \%)$ were screened and $18 \%$ screened positive. Less than $1 \%$ of eligible patients refused and $4 \%$ were not screened for reasons specified by MAs (refusal, acute medical illness, non-English speaking, blindness, deafness). Twenty-six percent of eligible patients were not screened with no reason reported; however, screened versus unscreened patients did not differ in age or proportions with a preexisting dementia diagnosis, specialty referral, or antidementia medication prescription (one-way ANOVA, all $P>0.10$ ).

\section{Impact of the intervention}

Clinic-level Outcomes: Change in Dementia Diagnoses, Specialty Referrals, and Medication Prescribing. In the baseline year, a dementia diagnosis was recorded for $2.6 \%$ of older patients in the control clinics and $4.1 \%$ of nongeriatricians' patients in the intervention clinics $(P=N S)$, with no differences between family practitioners and Internists. Conversely, geriatricians' patients were more than twice as likely as other physicians' patients to have an existing, baseline-year dementia diagnosis (10.8\%, $F=13.0, P=0.000, d f=2,1,913)$. In the whole 4clinic sample $(n=1,916)$, dementia diagnoses were strongly related to age $(<1 \%$ for patients $<70,1.8 \%$ at ages $70-74,2.4 \%$ at $75-79,9 \%$ at $80-84$, and $18 \%$ at $85+$ ). Dementia specialty referrals had been made in $<1 \%$ of the older population for all clinics at baseline, and, regardless of clinic or physician specialty, cholinesterase inhibitors were prescribed for about half of all patients with an existing dementia diagnosis.

Changes between baseline and intervention years are shown in Fig. 1. The proportion of patients with diagnosed dementia increased over time in both control and intervention clinics and for both geriatricians and nongeriatricians (Fig. 1a: $F=$ 14.52, $P=0.000, d f=1,360$, for geriatricians' patients; $F=6.08$, $P=0.014, d f=1,414$ for intervention clinic nongeriatricians' patients; $F=6.03, P=0.014, d f=1,1,139$ for control clinics). While percentages of nongeriatricians' patients diagnosed with dementia at baseline were similar for intervention and control clinics, percentages were significantly greater at endpoint in the intervention than control clinics $(F=4.87, P=0.027, d f=1$, $1,553)$, indicating an effect of the screening intervention. The intervention was also associated with a significant increase in dementia specialty referrals by nongeriatricians $(F=7.1, P=$ $0.008, d f=1,360$; Fig. 1b), to a level higher than control clinics $(F=10.8, P=0.001, d f=1,1,553)$ at the end of the intervention year. Referrals by intervention clinic geriatricians and by control clinic physicians did not change over time. Nongeria- 


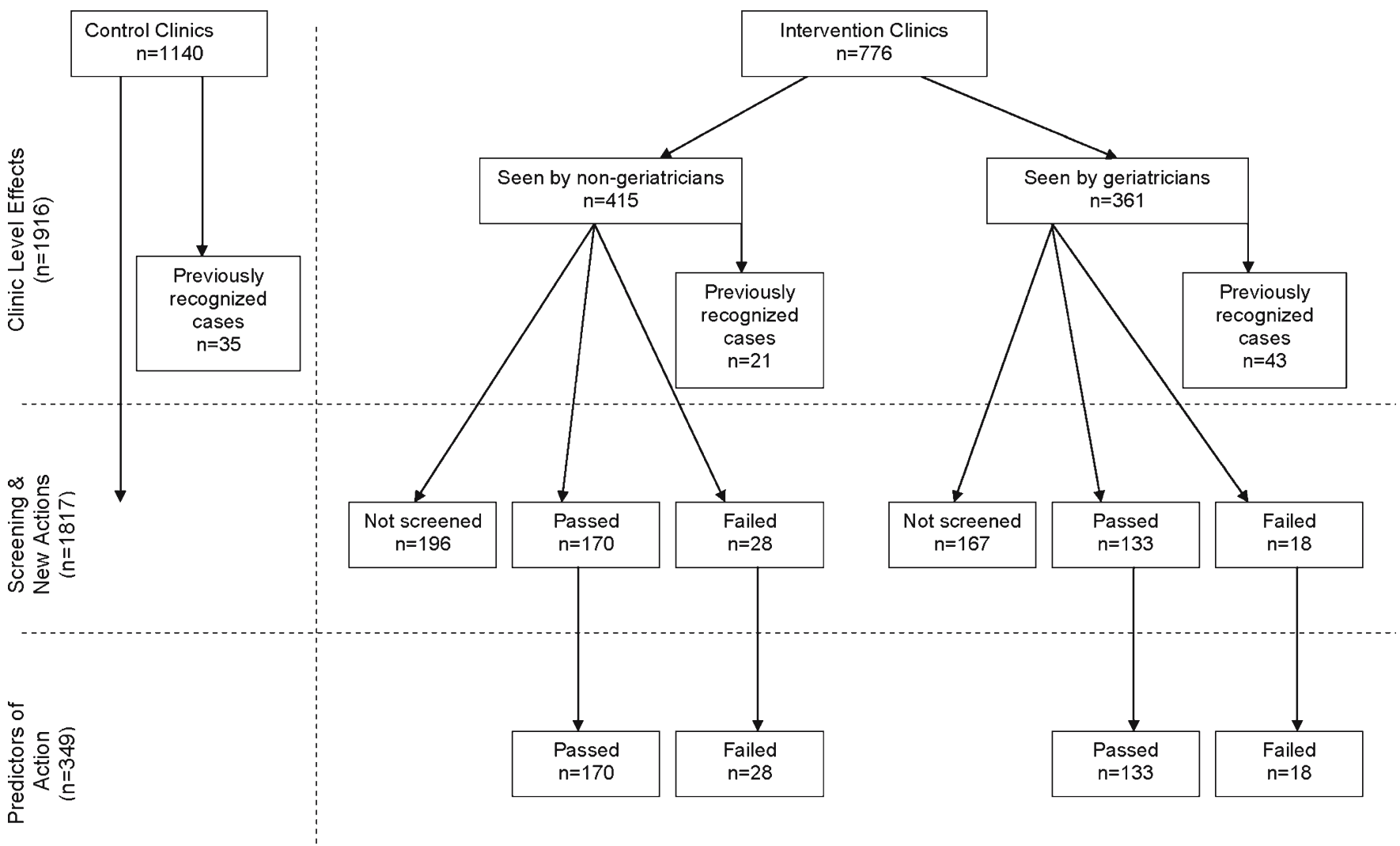

Figure 2. Derivation of sample for impact evaluation analyses

tricians increased their rates of prescribing of cholinesterase inhibitors in both intervention $(F=5.6, P=0.025, d f=1,360)$ and control $(F=4.0, P=0.045, d f=1,1,139)$ clinics (Fig. 1c), but geriatricians did not.

New Physician Actions Related to Screening. Using the composite indicator to assess impact in patients with no prior dementia indicators, evidence of new physician action was found in $17 \%(8 / 46)$ of patients with a positive screen and $1 \%$ $(3 / 303)$ of patients with a negative screen. A significant omnibus effect was found for screening (ANCOVAs controlling for age, across 7 previously defined subgroups; $F=14, d f=6$, 1,809; $P<0.001$; Fig. 3. Gender had no effect). Post hoc least significant difference tests revealed that control clinic patients had the lowest percentage of new physician actions (0.8\%). Significantly more physician actions were found for patients who failed the Mini-Cog than all other groups $(P<0.001)$, regardless of whether their physician was a geriatrician or not. Geriatricians took more dementia-related actions without screening than did nongeriatricians, but fewer than for screenpositive patients in either physician group $(P<0.05)$.

Similar results were obtained for individual dementia indicators, including rates of new dementia diagnoses (overall $F=16.0, d f=6,1,809, P<0.001)$ and new specialty referrals $(F=$ 14.7, $d f=6,1,809, P<0.001)$, with some differences between subgroups. Patients with positive screens were significantly more likely than all other groups to receive a new dementia diagnosis $(P<0.01)$ or specialty referral $(P<0.01)$. Referrals to a

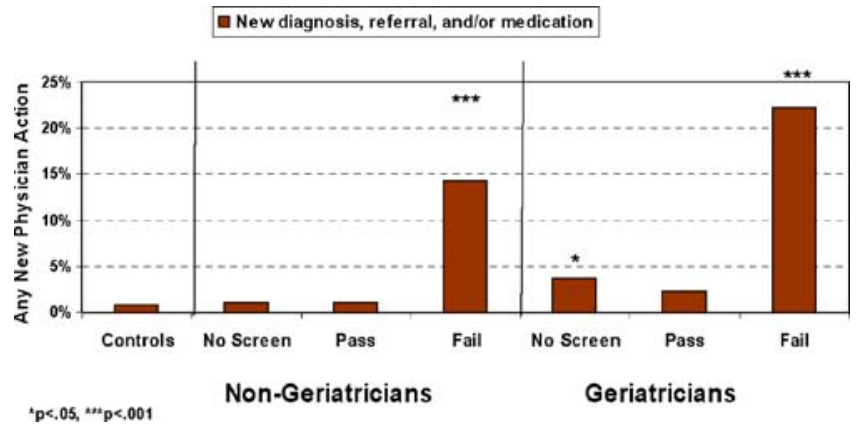

Figure 3. Impact of screening: patients without previous dementia indications 
dementia specialist were similar for geriatricians and nongeriatricians (5 vs 7\%). A small effect was seen for new medication prescriptions, evident only for screen-positive patients treated by nongeriatricians ( 3.6 vs $0.0-0.5 \%$ for all other groups, $F=$ 2.21, $d f=6,1,809, P=0.04)$.

Predictors of New Physician Actions. Using a composite indicator (any of the 3 target outcomes: diagnosis, referral, or medication, scored yes/no), logistic regression examined the influence of patient age, Mini-Cog score, and physician type (geriatrician vs nongeriatrician) on physician response among screened patients with no baseline dementia diagnosis. MiniCog score was the most important predictor (Wald statistic 16.9, Nagelkerke $R^{2}=35 \%, P=0.000$ ), followed by age (Wald statistic $=4.7$, Nagelkerke $R^{2}=6 \%, P=0.03$ ), with the strongest effect at the lowest scores. Post hoc inspection of physician response by screen score strongly favored the lowest and rarest Mini-Cog level in the failed range [Mini-Cog 0, 4 of 6 subjects (67\%) vs Mini-Cog 1-2, 4 of 40 (10\%)]. Physicians identified dementia in $1 \%$ of patients ( 3 of 303) who passed the Mini-Cog (scores 3-5). Older patient age was also positively associated with likelihood of a physician response $(0 \%$ of patients under age 75 vs $29 \%$ for patients 75 and older). In these analyses, physician specialty had no significant effect.

\section{DISCUSSION}

The first question was answered in the affirmative: cognitive screening was routinely and reliably implemented by nonphysician staff in primary care practices, using a very short, simple, validated screener, the Mini-Cog. Acceptability for both staff and patients was high, based on very low patient refusal rates and few complaints by medical assistants. Nevertheless, $26 \%$ of patients eligible for screening were not screened, with no specific reason recorded by the responsible MAs or the supervising RN. There could be many reasons for this, including aspects of clinic dynamics, individual MA factors, and patient factors. The data required to address all of the possible reasons why screening did not occur for $26 \%$ of eligible patients are relevant but unfortunately were not collected as part of this study. As reported in the "Results" section, age, gender, and prior dementia indicators (diagnoses, referrals, and cholinesterase inhibitor prescriptions) did not differ between screened and unscreened patients.

We found that the departure of the staff champion, whose role was to identify patients eligible for screening and to oversee the process, compromised the completion of the planned 1-year intervention. The electronic medical record used here was not programmed to allow automated flagging of patients eligible for screening, requiring time-consuming daily inspection by the nurse champion. A programmable electronic option could make the process proceed more efficiently.

The second question, whether instituting a clinic-wide screening policy with the Mini-Cog would lead to clinic-level changes in physician actions relevant to dementia, was also answered affirmatively. Relative to controls and negative screens, positive screens were associated with higher rates of dementia-related physician actions. Yet, we recognize that the magnitude of physician responses to screening as an isolated practice intervention, while statistically significant, was small relative to the frequency of screen failures. Physician response was identifiable for only $17 \%$ of patients who failed the MiniCog (but $0 \%$ of those under the age of 75). Primary care physicians acted mainly on positive screens when cognitive impairment was severe and only when patients were 75 years old or older. Although physician responses to positive screens were strikingly low, an independent German study of similar size, using the Mini-Mental Status Examination (MMSE) in primary care, ${ }^{14}$ suggests that this finding may be typical of current primary care practice. After being notified of previously undiagnosed patients who failed the MMSE, physicians in that study took further diagnostic or therapeutic steps in only $15 \%$ of cases.

We found little evidence that the clinic-wide screening intervention sensitized nongeriatricians to the possibility of cognitive impairment in their unscreened patients. By contrast, there was a small but significant increase in dementiarelated physician actions among geriatricians' patients who had not been screened. When age and Mini-Cog score were taken into account, differences in dementia-related actions between geriatricians and nongeriatricians disappeared, consistent with a presumption of geriatricians' greater awareness of cognitive impairment and a tendency for older, more demented patients to be treated by geriatricians when one is available. The addition of the Mini-Cog to primary care practice provides nongeriatricians with a tool that makes their behavior more similar to that of geriatricians with regard to dementia. ${ }^{1}$

The reasons for physicians' lack of response to a positive screen are unclear. Perhaps primary care physicians are reluctant to act on a positive screen in younger geriatric patients, particularly when cognitive disability is not severe. Perhaps younger patients who screened positive simply did not match physicians' expectations of how cognitively impaired patients "look," particularly if their mental image is of a frail older person with Alzheimer's disease. Other papers ${ }^{10,15,16}$ have discussed many possible explanations for physicians' reluctance to diagnose and treat dementia, such as concerns about labeling patients with a frightening disease diagnosis in the face of relatively weak medical treatment options, and obligating themselves to ordering an expensive, low-yield workup. However, these studies generally compare physicians' practices with research assessments rather than examining their responses when offered evidence of cognitive impairment through screening administered by staff of their own clinics as part of a routine medical visit.

Based on the present results and 1 prior study, ${ }^{14}$ it seems that physicians take a "wait-and-see" approach for the majority of patients with some evidence of cognitive impairment. Unfortunately, this approach tends to ignore cognitive impairment as A potential moderator of overall health care. It misses the opportunity to improve the general medical care of patients whose milder dementia might interfere with their ability to understand complex medical information and follow detailed medical regimens, including some diabetic, ${ }^{17}$ respiratory disease, ${ }^{18}$ hypertensive, ${ }^{19}$ and very old patients. ${ }^{20,21}$ Ignoring cognitive impairment until it is advanced also misses important opportunities to prevent high-cost, high-risk complications such as injury falls ${ }^{22}$ by anticipatory care management interventions.

There are several limitations of the present work. The completeness of outcome data obtained by the electronic 
record queries was not formally validated, nor were patients in this study evaluated for dementia using diagnostic interviews. The exclusive use of administrative data to track a limited range of physician responses could miss relevant qualitative information that could only be found in the detailed clinical record (e.g., further cognitive evaluation or a pertinent change in overall medical care plan). However, we found no such evidence in a review of 10 randomly selected medical records of patients who screened positive but did not receive a dementia diagnosis, referral, or prescription. Several of these patients made frequent visits to their physicians for minor complaints, used 1 or more medications known to impair cognition, or had unremarked weight loss, poorly controlled chronic diseases, or dehydration, all indirect indicators of possible cognitive impairment in older adults.

This is the first study we know of in which dementia screening has been instituted as a clinic-wide intervention, done by primary care staff themselves, as part of a "real-time" primary care practice. Whereas screening has been done in several prior studies of dementia in primary care, these efforts always involved research staff who were specifically trained for that single purpose. The advantages of our screening design include minimal additional burden on physician time, brief training required for reliable Mini-Cog screening by nonphysician staff, and no significant interruption of workflow.

\section{CONCLUSION}

Routine screening for dementia is the first step on a long road to optimal medical care for affected patients. A systematic review of studies in primary care from 1994 to $2002^{7}$ concluded that at least $50 \%$ of patients with frank dementia were undiagnosed, and that very mild dementia or mild cognitive impairment was diagnosed even less frequently. We have replicated this finding in a recent community study comparing physician diagnoses to standard research diagnostic assessment of dementia. ${ }^{1}$ That study demonstrated that the Mini-Cog detects most cases of mild dementia and many of mild cognitive impairment/very mild dementia, greatly improving on rates of recognition by patients' own physicians. ${ }^{1}$ Boustani et al., ${ }^{7}$ while acknowledging the paucity of data regarding the overall impact of dementia screening, argue that earlier identification of demented patients could potentially improve multiple outcomes for patients and their families.

It is evident that screening alone, while here shown to be relatively easy to implement with the Mini-Cog and to have positive impacts on physician actions, is insufficient to ensure optimal detection and management of cognitively impaired patients in primary care. It is well known that primary care screening for depression had no significant effect on patient outcomes until it was linked to effective management protocols. ${ }^{23}$ For dementia, making a diagnosis or referring the patient to a specialist is a necessary initial step but does not effectively represent the scope, process, or goals of dementia management. What is required is a paradigm shift.

The new paradigm would recognize that management of cognitively impaired older patients is best served by a chronic care model involving primary and specialty care, patients, families, and social service resources. Several models for delivering such care have recently been described. Two collaborative care management programs for patients already diag- nosed with dementia have been tested in primary care, one a randomized services trial utilizing a nurse manager in an academic primary care clinic, ${ }^{24}$ the other a randomized trial of dementia guideline education for physicians coupled with social service support. ${ }^{25}$ A third collaborative model, cooperative dementia group visits for patients and families, has been piloted with a group of medically complex dementia patients and their caregivers referred for ongoing specialty care. ${ }^{26}$ Pairing screening, which this study shows has a specific and positive impact on physician behavior, with formal dementia care management models could improve the rate at which physicians take action on a positive cognitive screen. Fostering primary care providers' awareness of, access to, and confidence in collaborative care approaches could then render dementia screening fully functional as a gateway to improving outcomes for patients.

Acknowledgments: This study was funded by an investigatorinitiated grant from Ortho McNeil (GAL-ALZ-419). Jürgen Unutzer MD, MPH, made valuable comments on the draft. Sherry Neher assisted with manuscript preparation.

Conflict of Interest: None disclosed.

Corresponding Author: Soo Borson, MD; Department of Psychiatry and Behavioral Sciences, University of Washington, 1959 NE Pacific Street, Campus Box 356560, Seattle, WA 98195, USA (e-mail: soob@u.washington.edu).

\section{REFERENCES}

1. Borson S, Scanlan JM, Watanabe J, Tu SP, Lessig M. Improving identification of cognitive impairment in primary care. Int $\mathrm{J}$ Geriatr Psychiatry. 2006;21:349-55.

2. Bush C, Kozak J, Elmslie T. Screening for cognitive impairment in the elderly. Can Fam Physician. 1997;43:1763-8.

3. Callahan CM, Hendrie HC, Tierney WM. Documentation and evaluation of cognitive impairment in elderly primary care patients. Ann Intern Med. 1995;122:422-9.

4. Evans DA, Funkenstein HH, Albert MS, et al. Prevalence of Alzheimer's disease in a community population of older persons. Higher than previously reported. JAMA. 1989;262:2551-6.

5. Valcour V, Masaki K, Curb J, Blanchette P. The detection of dementia in the primary care setting. Arch Intern Med. 2000;160:2964-8.

6. Ashford JW, Borson S, O'Hara R, et al. Should older adults be screened for dementia? Alzheimers Dement. 2006;2:76-85.

7. Boustani M, Peterson B, Hanson L, Harris R, Lohr KN. US Preventive Task Force. Screening for dementia in primary care: a summary of the evidence for the USPSTF. Ann Intern Med. 2003;138:927-37.

8. Boustani M, Callahan CM, Unverzagt FW, et al. Implementing a screening and diagnosis program for dementia in primary care. J Gen Intern Med. 2005;20:572-7.

9. Harvan JR, Cotter V. An evaluation of dementia screening in the primary care setting. J Am Acad Nurse Pract. 2006;18:351-60.

10. Boise L, Camicioli R, Morgan DL, Rose JH, Congelton L. Diagnosing dementia: perspectives of primary care physicians. Gerontologist. 1999;39:457-64.

11. Boise L, Neall MB, Kaye J. Dementia assessment in primary care: results from a study in three managed care systems. J Gerontol A Biol Sci Med Sci. 2004;59:M621-6.

12. Borson S, Scanlan J, Brush M, Vitaliano PP, Dokmak A. The Mini-Cog: A cognitive "vital signs" measure for dementia screening in multi-lingual elderly. Int J Geriatr Psychiatry. 2000;15:1021-7.

13. Borson S, Scanlan JM, Chen P, Ganguli M. The Mini-Cog as a screen for dementia: validation in a population-based sample. J Am Geriatr Soc. 2003;51:1451-4. 
14. Sandholzer H, Breull A, Fischer GC. Early diagnosis and early treatment of cognitive disorders: a study of geriatric screening of an unselected patient population in general practice. $Z$ Gerontol Geriatr. 1999;32:172-8.

15. Brodaty H, Howarth GC, Mant A, et al. General practice and dementia. A national survey of Australian GPs. Med J Aust. 2004;160:10-4.

16. Ganguli M, Rodriguez E, Mulsant B, et al. Detection and management of cognitive impairment in primary care: The Steel Valley Seniors Survey. $\mathrm{J}$ Am Geriatr Soc. 2004;52:1668-75.

17. Sinclair AJ, Girlin AJ, Bayer AJ. Cognitive dysfunction in older subjects with diabetes mellitus: impact on diabetes self-management and use of care services. Diabetes Res Clin Pract. 2000;50:203-12.

18. Allen SC, Jain M, Ragab S, Malik N. Acquisition and short-term retention of inhaler techniques require intact executive function in elderly subjects. Age Ageing. 2003;32:299-302.

19. Salas M, In't Veld BA, van der Linden PD, Hofman A, Breteler M, Stricker BH. Impaired cognitive function and compliance with antihypertensive drugs in elderly: the Rotterdam study. Clin Pharmacol Ther. 2001;70:561-6.
20. Royall DR, Cordes J, Polk M. Executive control and the comprehension of medical information by elderly retirees. Exp Aging Res. 1997;23:301-13.

21. Barat I, Andreasen F, Damsgaard EMS. Drug therapy in the elderly: what doctors believe and patients actually do. $\mathrm{Br} \mathrm{J}$ Clin Pharmacol. 2001;51:615-22.

22. Tinetti ME, Doucette J, Claus E, Marottoli R. Risk factors for serious injury during falls by older persons in the community. J Am Geriatr Soc. 1995;43:1214-21.

23. US Preventive Services Task Force. Screening for depression: recommendations and rationale. Ann Intern Med. 2002;136:760-4.

24. Callahan CM, Boustani MA, Unverzagt FW, et al. Effectiveness of collaborative care for older adults with Alzheimer's disease in primary care: a randomized controlled trial. JAMA. 2006;295:2148-57.

25. Vickrey B, Mittman BS, Connor KI, et al. The effect of a disease management intervention on quality and outcomes of dementia care: a randomized, controlled trial. Ann Intern Med. 2006;145:713-26.

26. Lessig M, Farrell J, Madhavan E, et al. Cooperative dementia care clinics: a new model for managing cognitively impaired patients. J Am Geriatr Soc. 2006;54:1937-42. 